\title{
Recurrent Painful Nodules Following Synthol Injection to Enhance Bicep Volume
}

Tudor Puiu, MD; Jesse Veenstra, MD, PhD; Albert S. Antonyan, MD; Alison Tisack, MD; Marsha Chaffins, MD

\section{PRACTICE POINTS}

- The use of injectable volumetric site enhancers in the form of oils to improve the aesthetic appearance of muscles has been prevalent for decades despite potentially serious adverse reactions.

- Complications from these procedures are underrecognized in the medical setting, perhaps owing to the trivialization of these procedures by the general public.

To the Editor:

A 28-year-old man presented to the dermatology clinic with red, tender, swollen nodules on the left arm of 5 days' duration, which had been a recurrent issue involving both arms. He also experienced intermittent fatigue and mild myalgia but denied associated fevers or chills. Oral clindamycin prescribed by a local emergency department provided some improvement. Upon further questioning, the patient admitted to injecting an unknown substance into the muscles 10 years prior for the purpose of enhancing their volume and appearance. Physical examination revealed large bilateral biceps with firm, mobile, nontender, subcutaneous nodules and mild erythema on the inner aspects of the arms. An incisional biopsy of a left arm nodule was performed with tissue culture
(Figure 1). Microscopic evaluation revealed mild dermal sclerosis with edema and sclerosis of fat septae (Figure 2A). The fat lobules contained granulomas with surrounding lymphocytes and clear holes noted within the histiocytic giant cells, indicating a likely foreign substance (Figure 2B). Immunohistochemical staining of the histiocytes with CD68 highlighted the clear vacuoles (Figure 3). Polarization examination, Alcian blue, periodic acid-Schiff, and acid-fast bacilli staining were negative. Bacterial, fungal, and mycobacterial tissue cultures and staining also were negative. The histologic findings of septal and lobular panniculitis with sclerosis and granulomatous inflammation in the clinical setting were consistent with a foreign body reaction secondary to synthol injection.

The willingness of athletes in competitive sports to undergo procedures or utilize substances for a competitive advantage despite both immediate and long-term consequences is well documented. ${ }^{1,2}$ In bodybuilding, use of anabolic steroids and intramuscular oil injections has been documented. ${ }^{3}$ The use of site enhancements in the form of "fillers" such as petroleum jelly and paraffin have been used for more than 100 years. ${ }^{4}$ The use of oil for volumetric site enhancement began in the 1960s in Italy with formebolone and evolved to the use of synthol in the 1990s. ${ }^{5}$ Synthol is a substance composed of $85 \%$ oil in the form of medium-chain triglycerides, $7.5 \%$ alcohol, and $7.5 \%$ lidocaine. ${ }^{6}$ The presumed mechanism of action of injected oils

Dr. Puiu is from the University of Michigan Medical School, Ann Arbor. Drs. Veenstra, Antonyan, Tisack, and Chaffins are from the Department of Dermatology, Henry Ford Hospital, Detroit, Michigan.

The authors report no conflict of interest.

Correspondence: Jesse Veenstra, MD, PhD, Henry Ford Health System, 3031 W Grand Blvd, Ste 800, Detroit, MI 48202 (jjveenst1@gmail.com). doi:10.12788/cutis.0189 


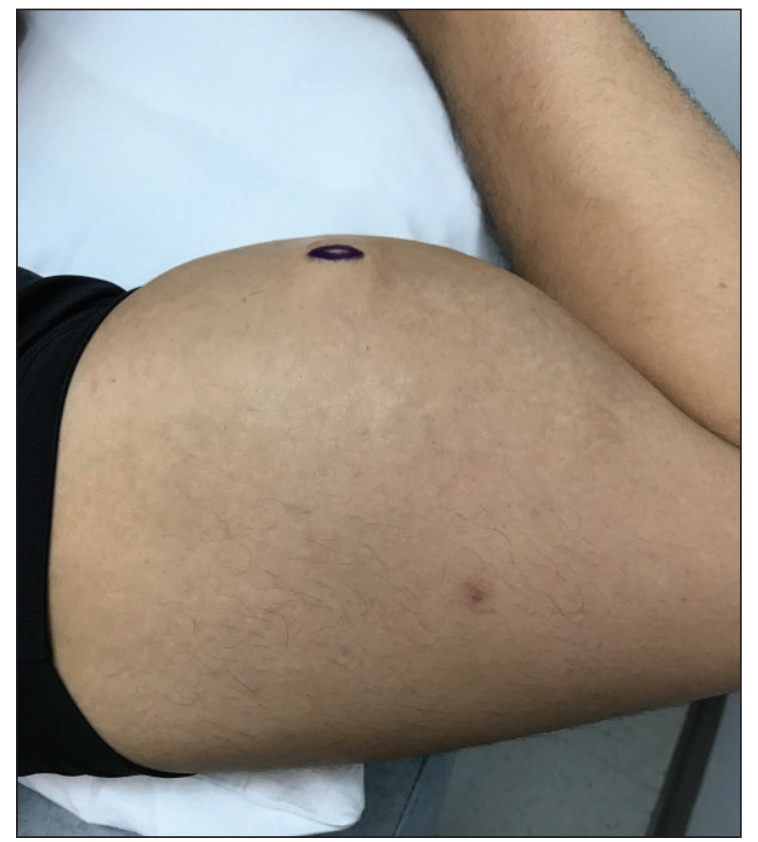

FIGURE 1. A disproportionately large left bicep relative to the patient's body habitus; the biopsy site is marked with ink. The patient reported injecting an unknown substance 10 years prior to enhance muscle volume.

consists of an initial inflammatory response followed by fibrosis and chronic macrophagocytosis, ultimately leading to expanded volume in the subcutaneous tissue.? These procedures are purely aesthetic with no increase in muscle strength or performance.

There are few cases in the literature of side effects from intramuscular synthol injections. In one report, a 29-yearold man presented with painful muscle fibrosis requiring open surgical excision of massively fibrotic bicep tissue. ${ }^{8}$ Another report documented a 45 -year-old man who presented with spontaneous ulcerations on the biceps that initially were treated with antibiotics and compression therapy but eventually required surgical intervention and skin grafting. ${ }^{9}$ Complications have been more frequently reported from injections of other oils such as paraffin and sesame. ${ }^{10,11}$ Given the similar underlying mechanisms of action, injected oils share the local side effects of inflammation, infection, chronic wounds, and ulceration,, 10 as well as a systemic risk for embolization leading to pulmonary emboli, myocardial infarction, and stroke. ${ }^{6}$ Although no standard of care exists for the management of complications arising from intramuscular oil injections, treatments that have been employed include antibiotics, corticosteroids, wound care, and compression therapy; definitive treatment typically is surgical excision. ${ }^{6,8,9,11,12}$ Psychiatric evaluation also should be considered to evaluate for the possibility of body dysmorphic disorder and other associated psychiatric conditions. ${ }^{11}$

Pressure for a particular aesthetic appearance, both within and outside the world of competitive sports,
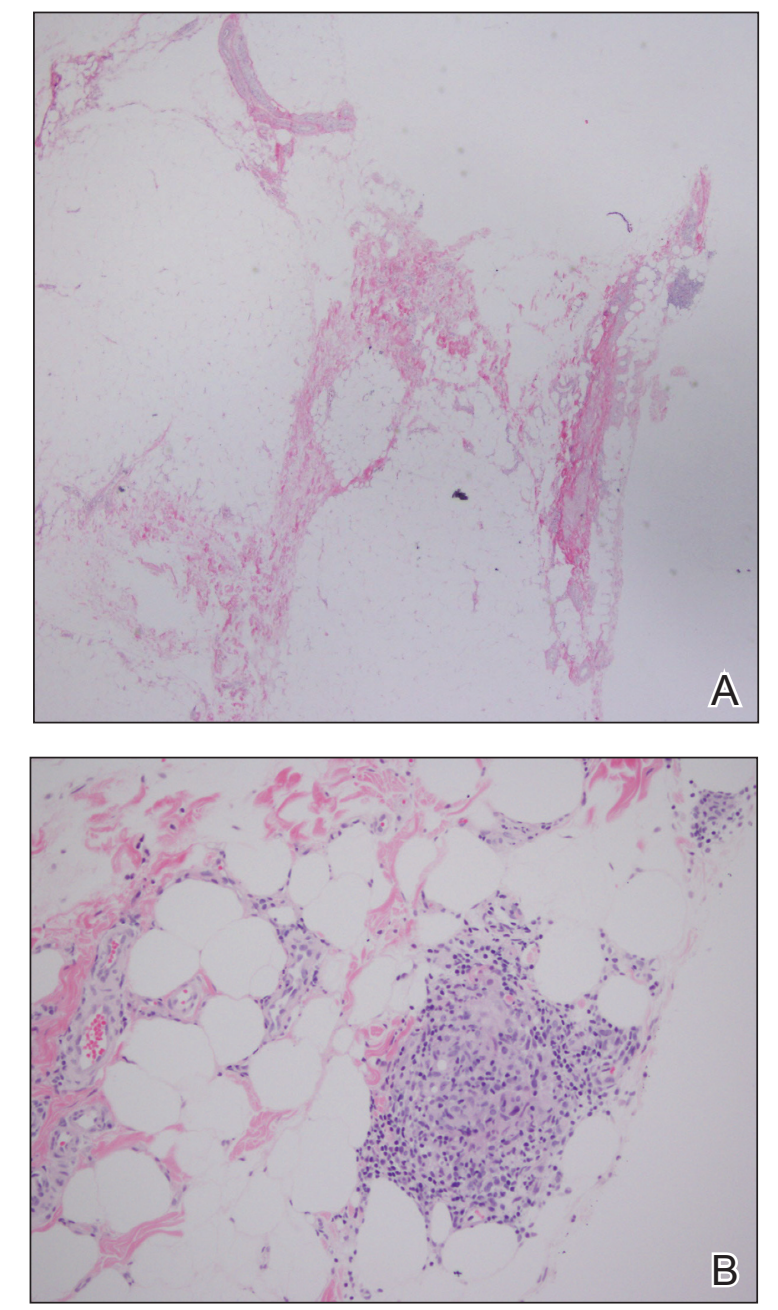

FIGURE 2. A, Histopathology demonstrated edema and sclerosis of fat septae with foci of granulomatous inflammation (H\&E, original magnification $\times 20$ ). B, High-power view of granulomatous inflammation with clear intracellular vacuoles noted within histiocytic giant cells, indicative of phagocytosis of foreign substance (H\&E, original magnification $\times 200)$.

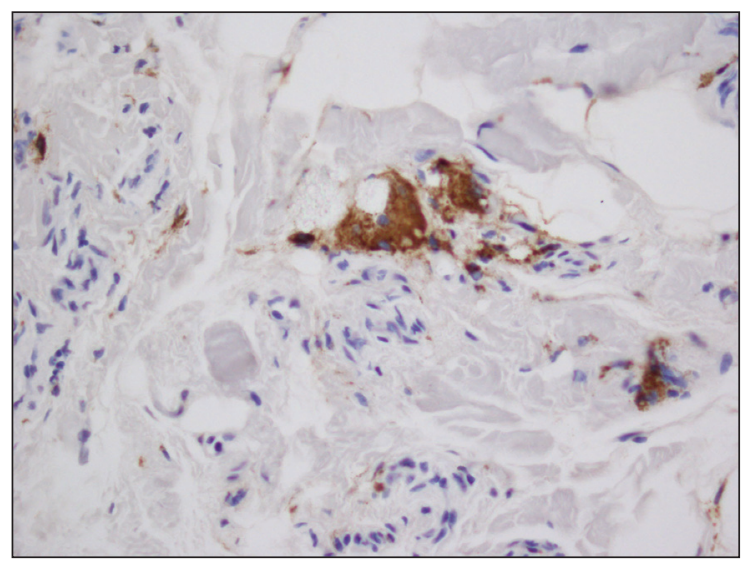

FIGURE 3. Immunohistochemistry showed CD68+ histiocytes containing clear intracellular vacuoles (original magnification $\times 200$ ). 
has driven individuals to various methods of muscular enhancement. Volumetric site enhancements have become increasingly popular, in part due to the perceived lack of systemic side effects, such as those associated with anabolic steroids. ${ }^{8}$ However, most users are unaware of the notable short-term and long-term risks associated with intramuscular oil injections. Synthol is widely available on the Internet and easily can be purchased and injected by anyone..$^{13}$ Medical providers should be aware of the possibility of aesthetic site enhancement use in their patients and be able to recognize and intervene in these cases to prevent chronic damage to muscle tissue and accompanying complications. Despite extensive commercialization of these products, few reports in the medical literature exist detailing the side effects of intramuscular oil injections, which may be contributing to the trivialization of these procedures by the general public. ${ }^{12}$

\section{REFERENCES}

1. Baron DA, Martin DM, Abol Magd S. Doping in sports and its spread to at-risk populations: an international review. World Psychiatry. 2007;6:118-123.

2. Holt RIG, Erotokritou-Mulligan I, Sönksen PH. The history of doping and growth hormone abuse in sport. Growth Horm IGF Res. 2009;19:320-326.
3. Figueiredo VC, Pedroso da Silva PR. Cosmetic doping-when anabolic-androgenic steroids are not enough. Subst Use Misuse. 2014;49:1163-1167.

4. Glicenstein J. The first "fillers," vaseline and paraffin. from miracle to disaster [in French]. Ann Chir Plast Esthet. 2007;52:157-161.

5. Evans NA. Gym and tonic: a profile of 100 male steroid users. Br J Sports Med. 1997;31:54-58.

6. Pupka A, Sikora J, Mauricz J, et al. The usage of synthol in the body building [in Polish]. Polim Med. 2009;39:63-65.

7. Di Benedetto G, Pierangeli M, Scalise A, et al. Paraffin oil injection in the body: an obsolete and destructive procedure. Ann Plast Surg. 2002;49:391-396.

8. Ghandourah S, Hofer MJ, Kiessling A, et al. Painful muscle fibrosis following synthol injections in a bodybuilder: a case report. J Med Case Rep. 2012;6:248.

9. Ikander P, Nielsen AM, Sørensen JA. Injection of synthol in a bodybuilder can cause chronic wounds and ulceration [in Danish]. Ugeskr Laeger. 2015;177:V12140642.

10. Henriksen TF, Løvenwald JB, Matzen SH. Paraffin oil injection in bodybuilders calls for preventive action [in Danish]. Ugeskr Laeger. 2010;172:219-220.

11. Darsow U, Bruckbauer H, Worret WI, et al. Subcutaneous oleomas induced by self-injection of sesame seed oil for muscle augmentation. J Am Acad Dermatol. 2000;42(2, pt 1):292-294.

12. Banke IJ, Prodinger PM, Waldt S, et al. Irreversible muscle damage in bodybuilding due to long-term intramuscular oil injection. Int J Sports Med. 2012;33:829-834.

13. Hall M, Grogan S, Gough B. Bodybuilders' accounts of synthol use: the construction of lay expertise online. J Health Psychol. 2016;21:1939-1948. 